\title{
Circulating tumour DNA abundance and potential utility in de novo metastatic prostate cancer
}

Gillian Vandekerkhove ${ }^{1 *}$, Werner J Struss ${ }^{{ }^{*}}$, Matti Annala ${ }^{2 *}$, Heini ML Kallio², Daniel

Khalaf ${ }^{3}$, Evan W Warner ${ }^{1}$, Cameron Herberts ${ }^{1}$, Elie Ritch ${ }^{1}$, Kevin Beja1 ${ }^{1}$ Yulia

Loktionova ${ }^{1}$, Antonio Hurtado-Coll1 ${ }^{1}$, Ladan Fazli1 ${ }^{1}$, Alan So ${ }^{1}$, Peter C Black ${ }^{1}$, Matti

Nykter2, Teuvo Tammela², Kim N Chi1,3, Martin E Gleave1, Alexander W Wyatt1

1Vancouver Prostate Centre, Department of Urologic Sciences, University of British Columbia, British Columbia, Canada; ${ }^{2}$ Prostate Cancer Research Center, Faculty of Medicine and Life Sciences and BioMediTech Institute, University of Tampere, Tampere, Finland; ${ }^{3}$ Department of Medical Oncology, British Columbia Cancer Agency.

${ }^{*}$ contributed equally

Correspondence to Dr. Alexander Wyatt, Vancouver Prostate Centre, Department of Urologic Sciences, University of British Columbia, 2660 Oak Street, Vancouver, BC, V6H 3Z6, Canada; Tel: +1-604-875-4818; Fax: +1-604-875-5654; Email:

awyatt@prostatecentre.com

Sequencing data was deposited to the European Genome-phenome Archive (EGA) under study identifier (EGAS00001003351).

Keywords: ctDNA, cfDNA, androgen deprivation therapy, castration-sensitive, cell-free DNA, DNA repair, liquid biopsy, precision oncology, sequencing, tissue biopsy

Word count abstract: 293

Word count text: 2592 


\section{Abstract}

Background: Several systemic therapeutic options exist for metastatic castratesensitive prostate cancer (mCSPC). Circulating tumour DNA (ctDNA) can molecularly profile metastatic castration-resistant prostate cancer (mCRPC) and can influence decision-making, but remains untested in $\mathrm{mCSPC}$.

Objective: To determine ctDNA abundance at de novo mCSPC diagnosis and whether ctDNA provides complementary clinically-relevant information to a prostate biopsy.

Design, Setting, and Participants: We collected plasma cell-free DNA (cfDNA) from 53 newly diagnosed patients with MCSPC and, where possible, during treatment. Targeted sequencing was performed on cfDNA and DNA from diagnostic prostate tissue.

Results and Limitations: Median ctDNA fraction was 11\% (range 0-84) among untreated patients but lower (1.0\%, range 0-51) in patients after short term (median 22 days) androgen deprivation therapy (ADT). TP53 mutations and DNA repair defects were identified in $47 \%$ and $21 \%$ of the cohort, respectively. Concordance for mutation detection in matched samples was $80 \%$. Combined ctDNA and tissue analysis identified potential driver alterations in $94 \%$ of patients, whereas ctDNA or prostate biopsy alone was insufficient in 19 cases (36\%). Limitations include the use of a narrow gene panel and undersampling of primary disease by prostate biopsy.

Conclusions: ctDNA provides additional information to a prostate biopsy in men with de novo mCSPC, but ADT rapidly reduces ctDNA availability. Primary tissue and ctDNA share relevant somatic alterations, suggesting that either are suitable for molecular subtyping in de novo mCSPC. The optimal approach for biomarker development should 
utilize both a tissue and liquid biopsy at diagnosis, as neither captures clinically-relevant somatic alterations in all patients.

Patient summary: In men with advanced prostate cancer, tumour DNA shed into the bloodstream can be measured by a blood test. The information from this test provides complementary information to a prostate needle biopsy and could be used to guide management strategies. 


\section{Introduction}

De novo metastatic disease represents $5-10 \%$ of prostate cancer (PCa) diagnoses but contributes to almost $50 \%$ of PCa related deaths [1,2]. The incidence of de novo metastatic diagnosis is rising, potentially related to improved imaging modalities and decreased prostate specific antigen (PSA) screening [3,4]. Historically, affected men were managed with systemic androgen deprivation therapy (ADT) alone, but recent phase III data supports treatment combination with taxane chemotherapy or androgen receptor (AR) targeted therapy in high burden disease [5-8]. Other targeted therapies such as poly (ADP-ribose) polymerase inhibitors (PARPi) are also being tested in metastatic castration-sensitive prostate cancer (mCSPC). As such, there is increasing interest in the potential for tumour molecular features to help guide therapy choice.

The majority of patients with de novo $\mathrm{mCSPC}$ will not undergo surgical management of their primary tumour, and metastatic biopsy is not routine. The only source of tissue is typically the diagnostic prostate biopsy. In some cases, diagnosis is based solely on clinical parameters such as exceptionally elevated PSA and concurrent radiographic bone lesions. Although next-generation sequencing of formalin-fixed paraffin-embedded (FFPE) tissue-derived DNA is now routine, it is unknown whether tumour cells obtained from prostate biopsy are representative of synchronous metastatic deposits.

Plasma circulating tumour DNA (ctDNA) is a promising minimally-invasive biomarker in progressing metastatic castration-resistant prostate cancer (mCRPC) [9-11]. The fraction of ctDNA as a proportion of total cell-free DNA (cfDNA) can approach $90 \%$ in 
mCRPC $[10,12]$. High ctDNA levels reflect proliferative disease and poor prognosis, and ctDNA-based mutational and copy number profiles are consistent with matched metastatic tissue $[10,12,13]$. However, plasma ctDNA is largely unexplored in mCSPC; it remains unclear whether acute ADT impacts ctDNA levels-a relevant question because de novo mCSPC patients may initiate ADT before the decision for treatment intensification (e.g. with chemotherapy). In this study, our objective was to determine ctDNA abundance at de novo mCSPC diagnosis and establish the degree to which molecular subtyping obtained from prostate biopsy tissue and ctDNA are complementary.

\section{Patients and Methods}

\section{Clinical cohort}

We prospectively enrolled 53 men diagnosed with de novo mCSPC at Vancouver General Hospital / University of British Columbia (UBC) Department of Urologic Sciences and British Columbia Cancer Agency from June 2014 to March 2018. A confirmatory transrectal ultrasound (TRUS) guided prostate biopsy was performed in 50 patients. Diagnoses were established by histology, PSA levels, and radiographic imaging (computed tomography and/or bone scan). All patients underwent blood collection for ctDNA analysis within 50 days of diagnosis. Where possible, blood was obtained at follow-up appointments. Three additional men with de novo mCSPC were enrolled at Tampere University Hospital from October 2017 to June 2018. Study approval was granted by the UBC Clinical Research Ethics Board (certificates H18-00944, H16-00934 and H09-01628) and the Regional Ethics Committee of 
Tampere University Hospital (certificate R03203). Written informed consent was obtained from all participants prior to enrollment.

\section{Sample processing, DNA sequencing, and bioinformatics}

Blood and tissue processing were performed as previously described (Supplementary Methods) $[10,14,15]$. We employed an established targeted sequencing strategy capturing the exons of $73 \mathrm{PCa}$ driver genes in cfDNA and tissue samples [10], modified by the inclusion of four bp molecular barcodes to the index sequence for cfDNA libraries. Sequence data analysis, including identification of somatic mutations and copy number alterations was performed according to published protocols [10]. ctDNA fraction was estimated based on somatic mutation allele fractions and leveraged matched tissue sample mutations in cases with low ctDNA fractions (Supplementary Methods). Deidentified sequencing data was deposited to the European Genome-phenome Archive (EGA) under study identifier (EGAS00001003351).

\section{Outcome measures}

Castration-resistance was defined according to Prostate Cancer Clinical Trials Working Group 3 guidelines [16]. Time to progression and follow-up were calculated from start of ADT. Survival fractions were estimated using the Kaplan-Meier method and differences between groups were identified using the logrank test. All hypothesis tests were twotailed and used a $5 \%$ significance threshold. Hazard ratios (HR) were calculated using 
Cox proportional hazards regression with binary covariates (dichotomized at cohort median), using "survival" package version 2.41.3 in R version 3.5.0. 


\section{Results}

Patient characteristics are provided in Table 1 and Supplementary Table 1. Plasma cfDNA sequencing was successful in 52/53 patients (median depth 927x;

Supplementary Table 2). 48/53 patients had diagnostic tissue available. Of the five patients without tissue, three had no local biopsy performed (clinical diagnoses only), while two had no remaining tumour after pathology slides were prepared. Tissue sequencing was successful in all 48 patients (median depth 189x).

\section{Androgen deprivation rapidly reduces ctDNA abundance}

For $35 / 53$ patients, plasma cfDNA was collected prior to ADT initiation; $74 \%(26 / 35)$ of these had detectable ctDNA (fraction range 2.0-84\%) (Fig. 1A; Supplementary Table 3), similar to the proportion of mCRPC patients that have detectable ctDNA with our approach [10,12]. 18 patients received 1-49 days of ADT (degarelix or goserelin plus bicalutamide) prior to cfDNA collection (median 22 days) (Fig. 1A); only 10 of 17 (59\%) with successfully sequenced cfDNA had detectable ctDNA, and ctDNA fractions were significantly lower than in treatment-naïve patients (mean $6.7 \%$ versus $23 \%$, median $1.0 \%$ versus $11 \% ; p=0.02$, ranksum test). The reduction in ctDNA fractions was more pronounced after one week of ADT.

For six patients with detectable ctDNA at diagnosis, we obtained follow-up plasma samples within four months of ADT initiation. In 5/6 patients, ctDNA was undetectable at follow-up (Fig. 1B). In one patient, ctDNA fraction increased from $50 \%$ to $70 \%$ between 
days 4 and 40 on ADT, despite a PSA decline. This patient subsequently began chemotherapy and ctDNA was undetectable in the third collection (102 days after ADT initiation). To confirm the overall trend, we examined serial samples from three patients collected within one week of commencing ADT. A clear reduction in ctDNA fraction was observed one day after ADT initiation. By day seven, ctDNA fractions were reduced to near zero (Fig. 1B; Supplementary Fig. 1).

Comprehensive diagnostic imaging data was available for 32 patients. All eight patients with liver or lung lesions (including three patients exposed to ADT) demonstrated detectable ctDNA, significantly higher than the remainder of the cohort with confirmed lymph node and/or bone metastases only (14/26, $p=0.03$, Fisher's exact test) (Fig. 1C). $3 / 8$ patients with visceral metastases had intraductal features in their prostate biopsy. We observed no relationship between ctDNA fraction and PSA, Gleason grade, or age (Fig. 1C).

\section{Aggressive genomic features with frequent TP53 mutations and DNA repair defects}

Combining somatic information from ctDNA and tumour tissue revealed a landscape similar to mCRPC [17], albeit without AR gene alterations (Fig. 2A; Supplementary Fig. 2; Supplementary Tables 4 and 5). TP53 mutations were identified in almost half the cohort (triple the frequency in localized disease [18]) while a further 11 patients without TP53 mutations harboured gene deletions. Eleven patients (21\%) exhibited 
truncating mutations within DNA damage repair (DDR) genes, including four patients with BRCA2 mutations (two germline). We identified two patients with CDK12 mutations and copy number profiles with multiple amplifications (e.g. CDK6, CCND1;

Supplementary Fig. 3) consistent with the CDK12-associated tandem duplication genotype $[19,20]$. We also identified truncating mutations in $R A D 51 C$ and $A T R$, but in neither case was deletion or mutation of the second allele evident (unlike all deleterious BRCA2 and ATM mutations; Supplementary Fig. 4). We identified one case with an MSH2 frameshift mutation (and deletion of the second allele) and a high tumour mutation burden consistent with mismatch repair (MMR) deficiency.

\section{ctDNA and tissue biopsy provide complementary insight to driver gene status}

Neither tumour tissue nor plasma cfDNA sequencing in isolation was sufficient to capture somatic information from all patients. We restricted analyses to the 35 patients with no prior $A D T$, thereby avoiding any confounding influence on ctDNA abundance. This subset included five patients (14\%) where either a tissue biopsy was not performed, or the biopsy core lacked somatic alterations (Fig. 2B). Importantly, in four of these patients somatic alterations were detected in ctDNA. There were also four ADTnaïve patients where, despite informative tumour tissue, the ctDNA fraction proved higher than the tumour tissue cellularity (as assessed by the same bioinformatic approach). Conversely, ten ADT-naïve samples had detectable ctDNA but at levels between 2 and $15 \%$, where low-level gene copy number changes are challenging to resolve. The majority of these patients had tumour tissue cellularity sufficient for copy 
number analysis (Fig. 2B). Finally, nine ADT-naïve samples had no ctDNA detected with our approach; tissue biopsy profiling better serves these patients. Across the entire cohort, no somatic information was obtained from either approach in only three cases (6\%). 2/3 patients had received prior ADT at time of cfDNA collection, compromising ctDNA abundance.

TP53 alterations are linked to poor prognosis and may represent an important variable to capture at initial diagnosis $[10,21]$. For ADT-naïve patients, over half $(9 / 17)$ of the non-silent TP53 mutations were missed by either tissue biopsy profiling or cfDNA sequencing, primarily due to failure of one approach to capture any somatic information, as described above (Fig. 2C). For DDR gene mutations, 9/13 were identified in both tissue and ctDNA (Fig. 2D). However, the MSH2 truncating mutation and accompanying hypermutation was only identified in the ctDNA of patient 11050; there was no evidence for this clone in matched tumour tissue, and the Gleason grade group of 1 suggests that the prostate biopsy undersampled disease. Three DDR gene mutations present in tumour tissue were not identified by cfDNA profiling; two alterations were in patients exposed to prior ADT at sample collection, confounding ctDNA detection. One patient with an ATM truncating mutation and monoallelic deletion in tissue had no detectable ctDNA despite being ADT-naïve and carrying a high plasma cfDNA concentration (16.7x cohort median). He had marrow infiltration and pancytopenia at time of blood collection, suggesting that ctDNA signal may have been diluted by elevated non-malignant cfDNA. 
It is unclear whether a primary tissue sample is representative of metastatic lesions in patients with de novo mCSPC. Here, mutational profiles of de novo mCSPC primary tissue and ctDNA were similar in cases where both approaches yielded sufficient tumour content for comparison. Among the 26 cases with somatic mutations detected in both tissue and ctDNA (excluding the MMR deficient case), 51/64 (80\%) were identified in both compartments (Supplementary Table 4; Supplementary Figs. 2, 5 and 6). Of the 13 mutations detected in only one sample, seven were unique to ctDNA, while six were found only in tissue.

\section{Majority of alterations in CSPC are shared at CRPC progression}

Follow-up for the cohort was 11 months. At time of writing, 18 patients had progressed with CRPC (including two with neuroendocrine PCa); this included 7/11 (63\%) patients harbouring DDR gene mutations (median time to progression 7.3 months $(95 \% \mathrm{Cl}$ : 3.2 18.7) compared to not reached ( $95 \% \mathrm{Cl}: 10.6$ - not reached) for the remainder of the cohort ( $p=0.01$, logrank test; Fig. 3A-B). Note that time to CRPC should be interpreted in the context of variable treatment regimens (Table 1). DDR gene status did not remain significant in multivariate analysis $(H R=2.21(0.77-6.37), p=0.1$; Supplementary Table 6), because PSA levels were higher in patients with DDR defects (median 290 versus $77 \mathrm{ng} / \mathrm{mL}, \mathrm{p}=0.005$, ranksum test).

For eleven patients, plasma cfDNA was collected after CRPC progression. 7/9 patients with detectable ctDNA post-progression developed either an $A R$ amplification or 
mutation (Fig. 4; Supplementary Table 4 and Supplementary Fig. 7). In general, few changes were detected outside of the $A R$, although one patient (who did not develop an $A R$ alteration) exhibited a hotspot CTNNB1 missense mutation at time of CRPC progression that was not identified in his diagnostic tissue sample (Fig. 4). Only one patient (17-111) demonstrated marked genomic differences between his diagnostic and CRPC specimens, however a shared PTEN stopgain mutation confirmed shared clonal ancestry. 


\section{Discussion}

Plasma ctDNA is abundant in most patients with treatment-naïve de novo mCSPC, providing additional insight into metastatic disease beyond that available from prostate biopsy. However, ADT exposure prior to blood collection significantly reduced ctDNA abundance, thereby impairing detection of clinically-relevant somatic alterations. Since ctDNA originates from apoptosis of cancer cells [22,23], a transient spike in ctDNA fractions a few hours after therapy initiation remains possible. Furthermore, ADT type (e.g. degarelix versus goserelin) differentially impacts the rate at which castrate testosterone is achieved, and may be related to the rate of ctDNA decline. We have also not assessed whether the biopsy procedure impacts ctDNA or non-malignant cfDNA release. Nevertheless, our data suggests that in order for ctDNA to guide treatment intensification in mCSPC, blood collection timing (relative to ADT initiation) warrants careful consideration.

Particularly high ctDNA levels were observed in patients with visceral metastases, consistent with mCRPC where ctDNA fractions correlate with clinical prognostic markers [9-11]. Therefore, clinical metrics of proliferative tumour volume may help guide implementation of ctDNA assays in mCSPC. Also similar to mCRPC [12], somatic mutations identified in ctDNA were highly concordant with matched tissue biopsies. However, while there were cases where ctDNA proved more informative than tissue biopsy (for detection of driver gene alterations), the opposite was also true as some patients had low ctDNA levels. Technological advances continue to improve detection sensitivity for ultra-rare mutations in cfDNA [24], but common PCa copy number 
alterations such as PTEN or CHD1 deletion remain undetectable when ctDNA constitutes a few percent of total cfDNA. Many of the alterations identified by either cfDNA or tissue sequencing alone have clinical relevance, from DDR gene defects and potential sensitivity to PARPi or immunotherapy $[25,26]$, to TP53 and SPOP mutations that infer poor and favourable prognosis, respectively $[10,27,28]$. Therefore, the optimal approach for correlative studies or biomarker development in the de novo mCSPC setting should incorporate both tissue and plasma analyses, or risk undersampling disease.

De novo $\mathrm{mCSPC}$ is poorly characterized since sequencing efforts have focused on either localized disease or mCRPC. In our study, the similarity between primary tissue and ctDNA may suggest that de novo mCSPC is a highly clonal disease at diagnosis, although follow-up studies are required to confirm this hypothesis. In localized PCa, intra-tumour heterogeneity is common, and truly independent tumour foci can arise within the same prostate $[29,30]$. It is possible that de novo mCSPC represents later stage disease, after the most aggressive tumour clone expands and predominates. Alternatively, it may represent a different disease trajectory, characterized by emergence of a singularly aggressive clone that rapidly proliferates. Regardless, de novo $\mathrm{mCSPC}$ is characterized by aggressive genomics including frequent TP53 and DDR gene mutations; this appears distinct from localized disease, but different sequencing and analysis approaches between studies prevent definitive conclusions. Among patients who progressed to CRPC, ctDNA at progression yielded highly similar profiles to their CSPC counterpart, suggesting that de novo mCSPC is primed for 
therapy resistance. Future studies assessing larger patient numbers and a broader range of somatic alterations are required.

To maintain cost-efficiency, we captured a small fraction of the genome and did not perform ultra-deep sequencing (i.e. $\sim 10,000 x$ ). Some samples with apparent low tumour content may harbour somatic alterations at high variant frequency outside the panel, or conversely harbour alterations below our detection sensitivity. The unavoidable sampling bias associated with TRUS-guided needle biopsy may account for mutations detected only in ctDNA. Future studies could instead assess saturation template biopsies. Finally, given the level of noise associated with FFPE tissue-derived copy number profiles, comparisons with ctDNA-derived copy number alterations were limited.

\section{Conclusions}

Plasma ctDNA fractions are elevated in de novo mCSPC, especially in patients with visceral metastases. However, exposure to ADT compromises the potential utility of ctDNA. When measurable, ctDNA defines the driver alterations in de novo mCSPC, but combined use of ctDNA and primary tissue is optimal for assessing molecular subtype and could aid targeted therapy implementation in a precision oncology framework. 


\section{Acknowledgements}

This work was supported by a Canadian Institutes of Health Research (CIHR) project grant (KC, AW), the Prostate Cancer Foundation (KC, AW), Prostate Cancer Canada through the Movember Rising Star in Prostate Cancer research program (AW), Academy of Finland (MA, MN), Business Finland (HK), and a Terry Fox New Frontiers Program Project grant \#TFF116129 (KC, MG, AW). The authors wish to acknowledge CSC - IT Center for Science, Finland, for computational resources. 


\section{References}

[1] Helgstrand JT, Røder MA, Klemann N, Toft BG, Lichtensztajn DY, Brooks JD, et al. Trends in incidence and 5-year mortality in men with newly diagnosed, metastatic prostate cancer-A population-based analysis of 2 national cohorts. Cancer 2018. doi:10.1002/cncr.31384.

[2] Buzzoni C, Auvinen A, Roobol MJ, Carlsson S, Moss SM, Puliti D, et al. Metastatic Prostate Cancer Incidence and Prostate-specific Antigen Testing: New Insights from the European Randomized Study of Screening for Prostate Cancer. Eur Urol 2015;68:885-90.

[3] Weiner AB, Matulewicz RS, Eggener SE, Schaeffer EM. Increasing incidence of metastatic prostate cancer in the United States (2004-2013). Prostate Cancer Prostatic Dis 2016;19:395-7.

[4] Hu JC, Nguyen P, Mao J, Halpern J, Shoag J, Wright JD, et al. Increase in Prostate Cancer Distant Metastases at Diagnosis in the United States. JAMA Oncol $2017 ; 3: 705-7$.

[5] Sweeney CJ, Chen Y-H, Carducci M, Liu G, Jarrard DF, Eisenberger M, et al. Chemohormonal Therapy in Metastatic Hormone-Sensitive Prostate Cancer. N Engl J Med 2015;373:737-46.

[6] Fizazi K, Tran N, Fein L, Matsubara N, Rodriguez-Antolin A, Alekseev BY, et al. Abiraterone plus Prednisone in Metastatic, Castration-Sensitive Prostate Cancer. N Engl J Med 2017;377:352-60.

[7] James ND, Sydes MR, Clarke NW, Mason MD, Dearnaley DP, Spears MR, et al. Addition of docetaxel, zoledronic acid, or both to first-line long-term hormone 
therapy in prostate cancer (STAMPEDE): survival results from an adaptive, multiarm, multistage, platform randomised controlled trial. Lancet 2016;387:116377.

[8] James ND, de Bono JS, Spears MR, Clarke NW, Mason MD, Dearnaley DP, et al. Abiraterone for Prostate Cancer Not Previously Treated with Hormone Therapy. N Engl J Med 2017;377:338-51.

[9] Romanel A, Gasi Tandefelt D, Conteduca V, Jayaram A, Casiraghi N, Wetterskog D, et al. Plasma AR and abiraterone-resistant prostate cancer. Sci Transl Med 2015;7:312re10.

[10] Annala M, Vandekerkhove G, Khalaf D, Taavitsainen S, Beja K, Warner EW, et al. Circulating Tumor DNA Genomics Correlate with Resistance to Abiraterone and Enzalutamide in Prostate Cancer. Cancer Discov 2018;8:444-57.

[11] Belic J, Graf R, Bauernhofer T, Cherkas Y, Ulz P, Waldispuehl-Geigl J, et al. Genomic alterations in plasma DNA from patients with metastasized prostate cancer receiving abiraterone or enzalutamide. International Journal of Cancer 2018;143:1236-48.

[12] Wyatt AW, Annala M, Aggarwal R, Beja K, Feng F, Youngren J, et al. Concordance of Circulating Tumor DNA and Matched Metastatic Tissue Biopsy in Prostate Cancer. J Natl Cancer Inst 2017;109. doi:10.1093/jnci/djx118.

[13] Hovelson DH, Liu C-J, Wang Y, Kang Q, Henderson J, Gursky A, et al. Rapid, ultra low coverage copy number profiling of cell-free DNA as a precision oncology screening strategy. Oncotarget 2017;8:89848-66.

[14] Beltran H, Wyatt AW, Chedgy EC, Donoghue A, Annala M, Warner EW, et al. Impact 
of Therapy on Genomics and Transcriptomics in High-Risk Prostate Cancer Treated with Neoadjuvant Docetaxel and Androgen Deprivation Therapy. Clin Cancer Res 2017;23:6802-11.

[15] Chedgy EC, Vandekerkhove G, Herberts C, Annala M, Donoghue AJ, Sigouros M, et al. Biallelic tumor suppressor loss and DNA repair defects in de novo small cell prostate cancer. J Pathol 2018. doi:10.1002/path.5137.

[16] Scher HI, Morris MJ, Stadler WM, Higano C, Basch E, Fizazi K, et al. Trial Design and Objectives for Castration-Resistant Prostate Cancer: Updated Recommendations From the Prostate Cancer Clinical Trials Working Group 3. J Clin Oncol 2016;34:1402-18.

[17] Robinson D, Van Allen EM, Wu Y-M, Schultz N, Lonigro RJ, Mosquera J-M, et al. Integrative clinical genomics of advanced prostate cancer. Cell 2015;161:1215-28.

[18] Cancer Genome Atlas Research Network. The Molecular Taxonomy of Primary Prostate Cancer. Cell 2015;163:1011-25.

[19] Wu Y-M, Cieślik M, Lonigro RJ, Vats P, Reimers MA, Cao X, et al. Inactivation of CDK12 Delineates a Distinct Immunogenic Class of Advanced Prostate Cancer. Cell 2018;173:1770-82.e14.

[20] Quigley DA, Dang HX, Zhao SG, Lloyd P, Aggarwal R, Alumkal JJ, et al. Genomic Hallmarks and Structural Variation in Metastatic Prostate Cancer. Cell 2018;174:758-69.e9.

[21] Maughan BL, Guedes LB, Boucher K, Rajoria G, Liu Z, Klimek S, et al. p53 status in the primary tumor predicts efficacy of subsequent abiraterone and enzalutamide in castration-resistant prostate cancer. Prostate Cancer Prostatic Dis 2018;21:260- 
8.

[22] Volik S, Alcaide M, Morin RD, Collins C. Cell-free DNA (cfDNA): Clinical

Significance and Utility in Cancer Shaped By Emerging Technologies. Mol Cancer Res 2016;14:898-908.

[23] Corcoran RB, Chabner BA. Application of Cell-free DNA Analysis to Cancer Treatment. N Engl J Med 2018;379:1754-65.

[24] Newman AM, Lovejoy AF, Klass DM, Kurtz DM, Chabon JJ, Scherer F, et al. Integrated digital error suppression for improved detection of circulating tumor DNA. Nat Biotechnol 2016;34:547-55.

[25] Mateo J, Carreira S, Sandhu S, Miranda S, Mossop H, Perez-Lopez R, et al. DNARepair Defects and Olaparib in Metastatic Prostate Cancer. N Engl J Med 2015;373:1697-708.

[26] Nava Rodrigues D, Rescigno P, Liu D, Yuan W, Carreira S, Lambros MB, et al. Immunogenomic analyses associate immunological alterations with mismatch repair defects in prostate cancer. J Clin Invest 2018. doi:10.1172/JCI121924.

[27] De Laere B, Oeyen S, Mayrhofer M, Whitington T, van Dam P-J, Van Oyen P, et al. TP53 outperforms other androgen receptor biomarkers to predict abiraterone or enzalutamide outcome in metastatic castration-resistant prostate cancer. Clin Cancer Res 2018:clincanres.1943.2018.

[28] Boysen G, Rodrigues DN, Rescigno P, Seed G, Dolling D, Riisnaes R, et al. SPOPMutated/CHD1-Deleted Lethal Prostate Cancer and Abiraterone Sensitivity. Clin Cancer Res 2018. doi:10.1158/1078-0432.CCR-18-0937.

[29] Boutros PC, Fraser M, Harding NJ, de Borja R, Trudel D, Lalonde E, et al. Spatial 
genomic heterogeneity within localized, multifocal prostate cancer. Nat Genet 2015;47:736-45.

[30] Løvf M, Zhao S, Axcrona U, Johannessen B, Bakken AC, Carm KT, et al. Multifocal Primary Prostate Cancer Exhibits High Degree of Genomic Heterogeneity. Eur Urol 2018. doi:10.1016/j.eururo.2018.08.009. 


\section{Tables and Figures}

Median age at diagnosis (IQR)

Median PSA at diagnosis (IQR)

Gleason grade group

1

2

3

4

5

Unknown

Metastatic extent of disease at diagnosis

Lymph node only

Regional

Non-regional

Bone

Lung

Liver

Initial therapy regimen post-diagnosis

ADT only

ADT + docetaxel (without AR targeted therapy)

ADT + AR targeted therapy*

ADT + docetaxel + AR targeted therapy*

Unknown**

Patients with cfDNA collected prior to ADT initiation

Patients with cfDNA collected post ADT initiation (range in days)

Table 1. Clinical characteristics at diagnosis with de novo metastatic castration-sensitive prostate cancer (mCSPC). IQR = interquartile range; $A D T$ = androgen deprivation therapy, $A R=$ androgen receptor. *Patients enrolled in a blinded study.
$14(26 \%)$

$18(34 \%)$

$68(60-76)$

$110(32-280)$

$1(2 \%)$

$0(0 \%)$

$3(6 \%)$

$6(11 \%)$

$38(72 \%)$

$5(9 \%)$

$5(9 \%)$

2

3

40 (75\%)

$6(11 \%)$

$2(4 \%)$

$9(17 \%)$

$8(15 \%)$

$4(8 \%)$

$67 \%$

$33 \%(1-49)$ 


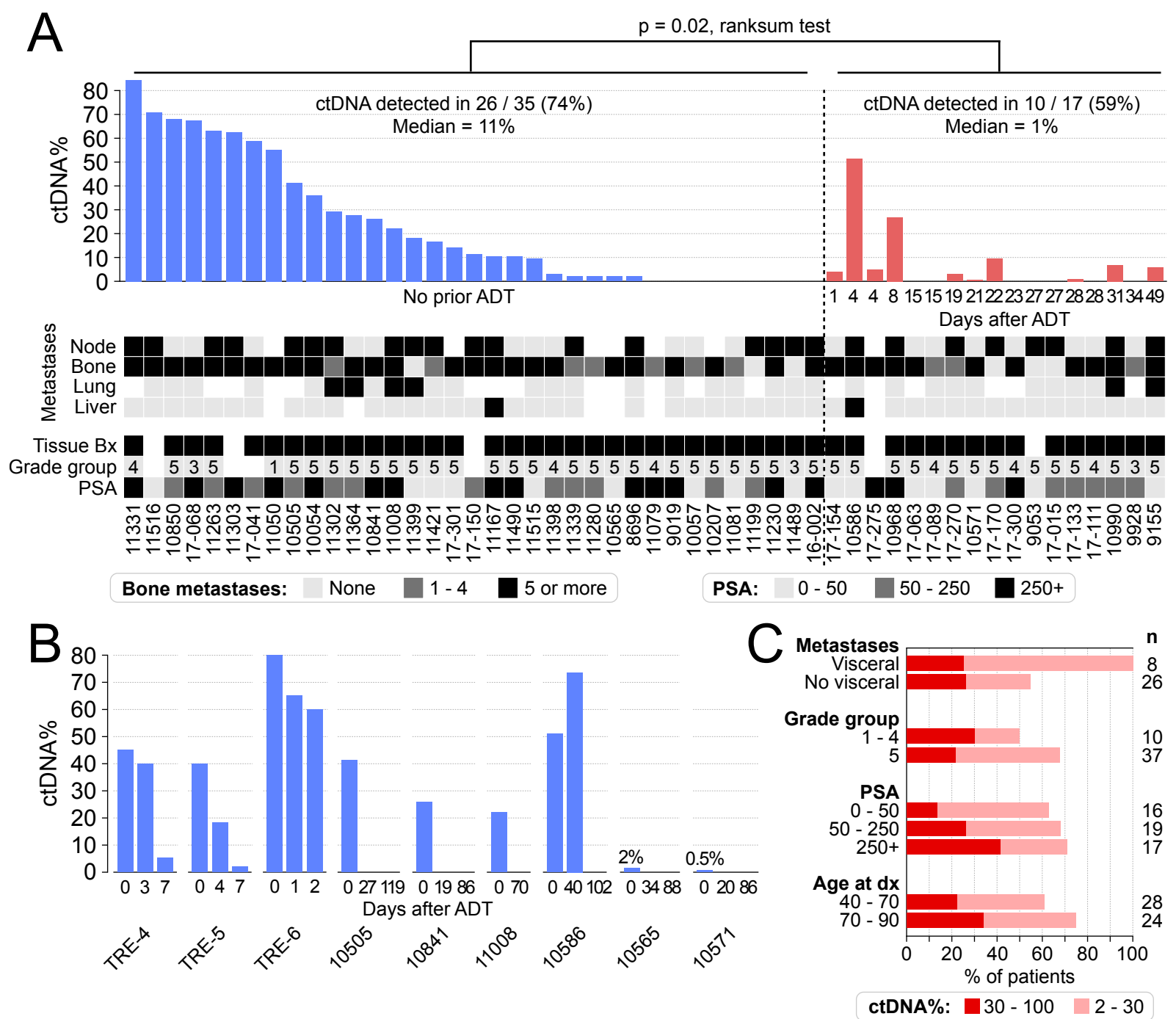

Figure 1. Circulating tumour DNA (ctDNA) abundance and impact of androgen deprivation therapy (ADT). A) Bar plot illustrating the percentage of cell-free DNA (cfDNA) that is tumour-derived (i.e. the ctDNA\%) for each patient. Blue bars reflect patients that were entirely treatment-naïve at time of blood collection; red bars indicate those exposed to short term ADT. Key clinical variables at diagnosis are indicated in the matrix below the bar plot. B) Bar plot illustrating ctDNA fraction decline in serial blood collections after commencement of ADT (see also Supplementary Fig. 1). C) Proportion of patients with detected ctDNA, based on clinical variables. PSA = prostate specific antigen; $\mathrm{Bx}=$ biopsy; $\mathrm{dx}=$ diagnosis. 


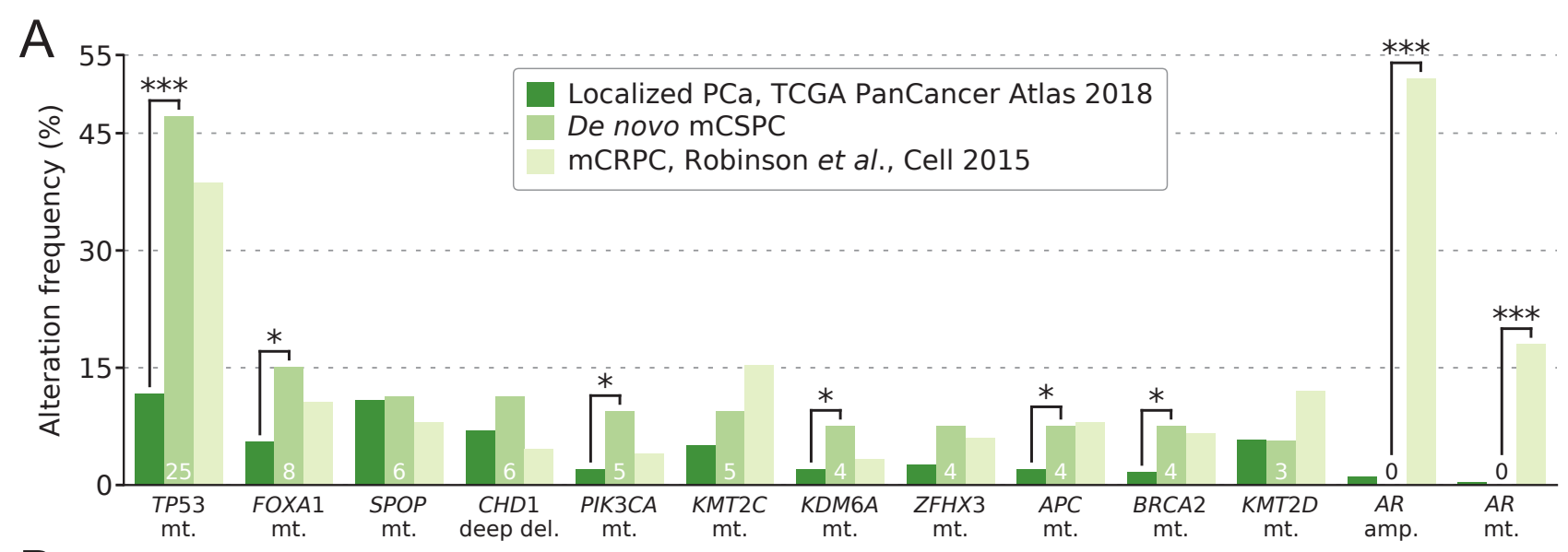

B
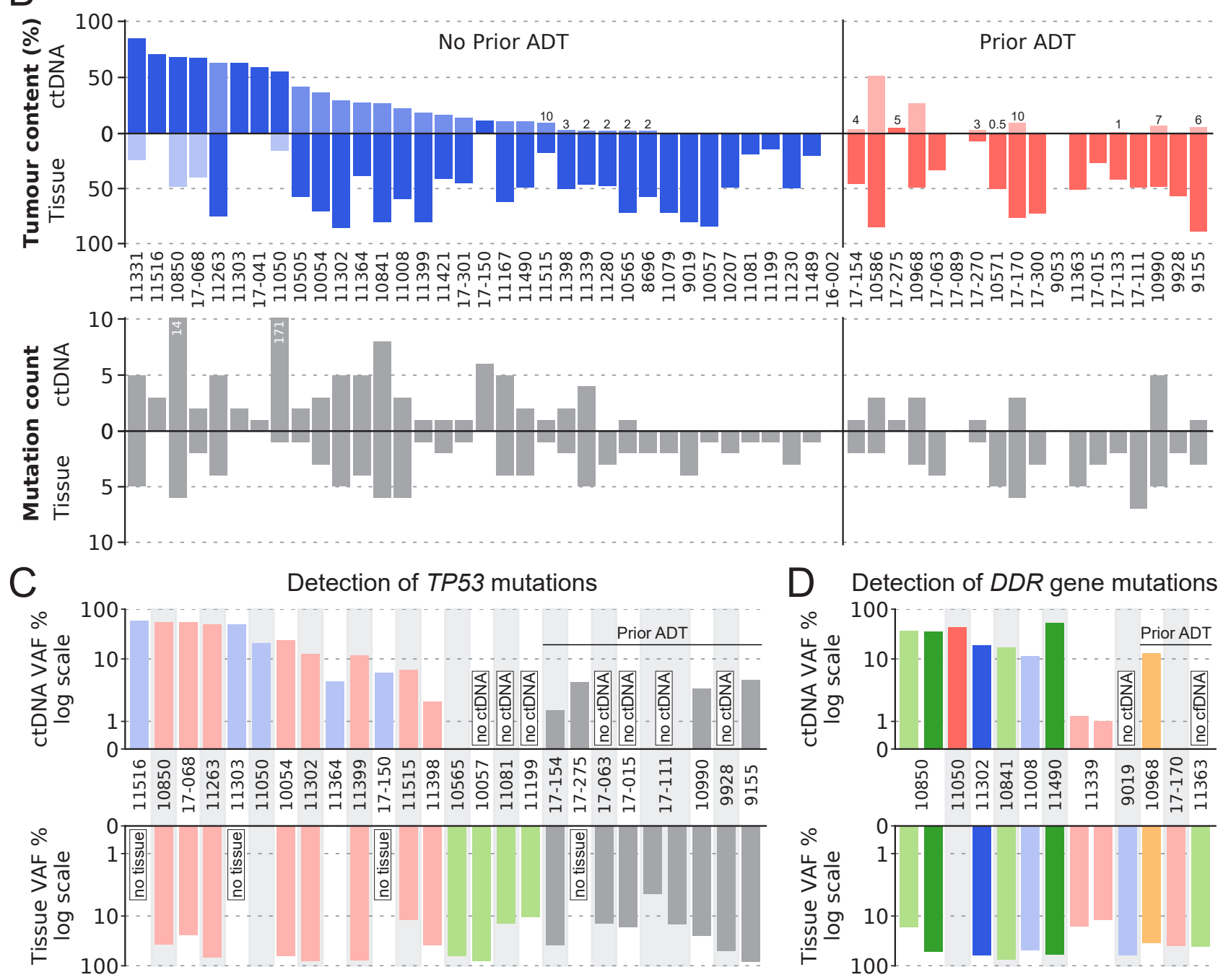

TP53 mutation detection in patients without prior ADT:

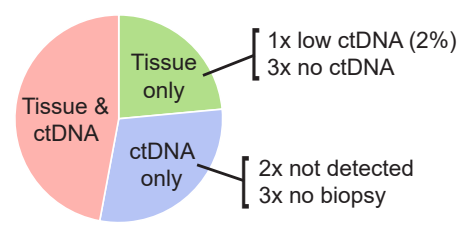

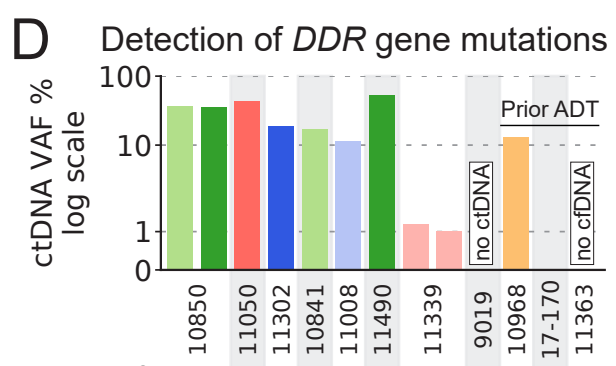

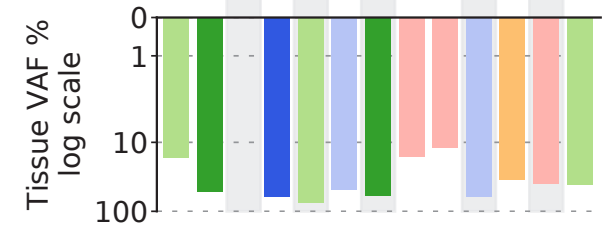

D Detection of $D D R$ gene mutations

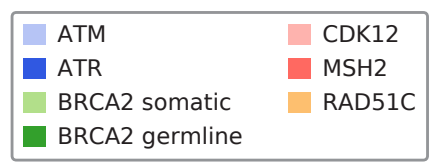


Figure 2. Combined analysis of circulating tumour DNA (ctDNA) and primary tissue reveals aggressive genomic features. A) Frequency of recurrent somatic alterations in de novo metastatic castration-sensitive prostate cancer (mCSPC) as compared to localized prostate cancer ( $\mathrm{PCa}$ ) and metastatic castration-resistant prostate cancer (mCRPC). Note that sequencing platforms and bioinformatic approaches differ between each study, limiting the conclusions that can be drawn by study-to-study comparison. B) Bar plots demonstrating tumour content in tissue compared to matched ctDNA (upper panel), and somatic mutation count as derived from these samples (lower panel); stratified by exposure to androgen deprivation therapy (ADT). C) Concordance of TP53 mutation detection between matched samples. Pie chart indicates proportion of TP53 mutations detected by each assay in ADT-naïve patients. D) Concordance of DNA damage repair $(\mathrm{DDR})$ gene calls. $\mathrm{mt} .=$ mutation; deep del. $=$ deep deletion; amp. $=$ amplification. 


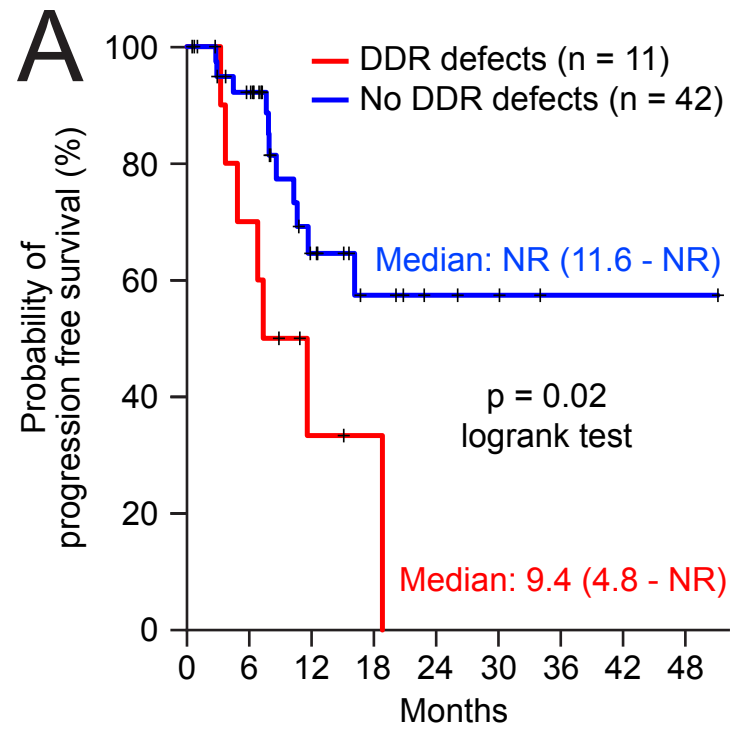

C

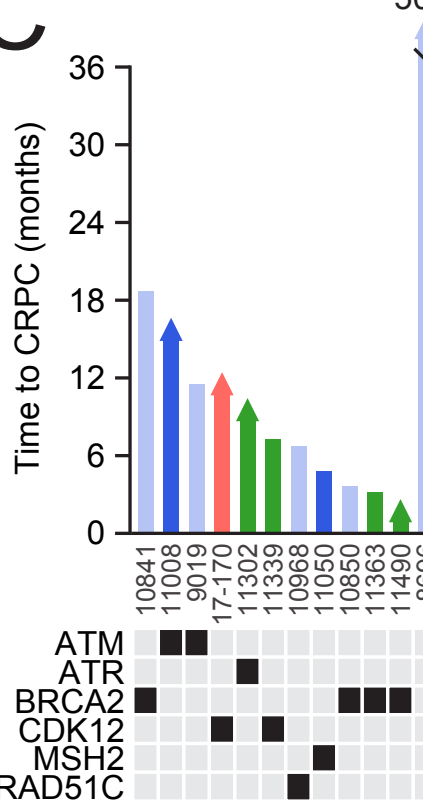

50.8
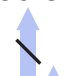

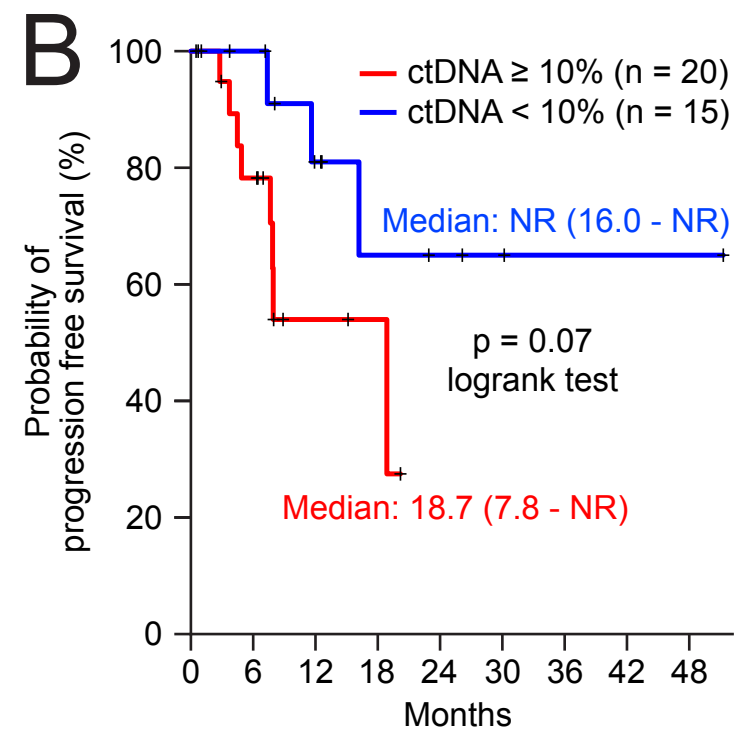

\begin{tabular}{|l} 
ADT \\
ADT+AR \\
ADT+DOC \\
ADT+DOC+AR \\
Unknown
\end{tabular}

Unknown

Figure 3. DNA damage repair (DDR) gene defects are associated with earlier

progression to castration-resistant prostate cancer (CRPC). A) Kaplan-Meier plot of time to CRPC from ADT initiation in patients with and without deleterious DDR gene

alterations. B) Kaplan-Meier plot showing the association of ctDNA fraction and time to CRPC from ADT initiation. C) Swimmers plot of time to CRPC progression from diagnosis, stratified by DDR gene status. ADT = androgen deprivation therapy; $A R=$ androgen receptor targeted therapy; DOC = docetaxel chemotherapy. 


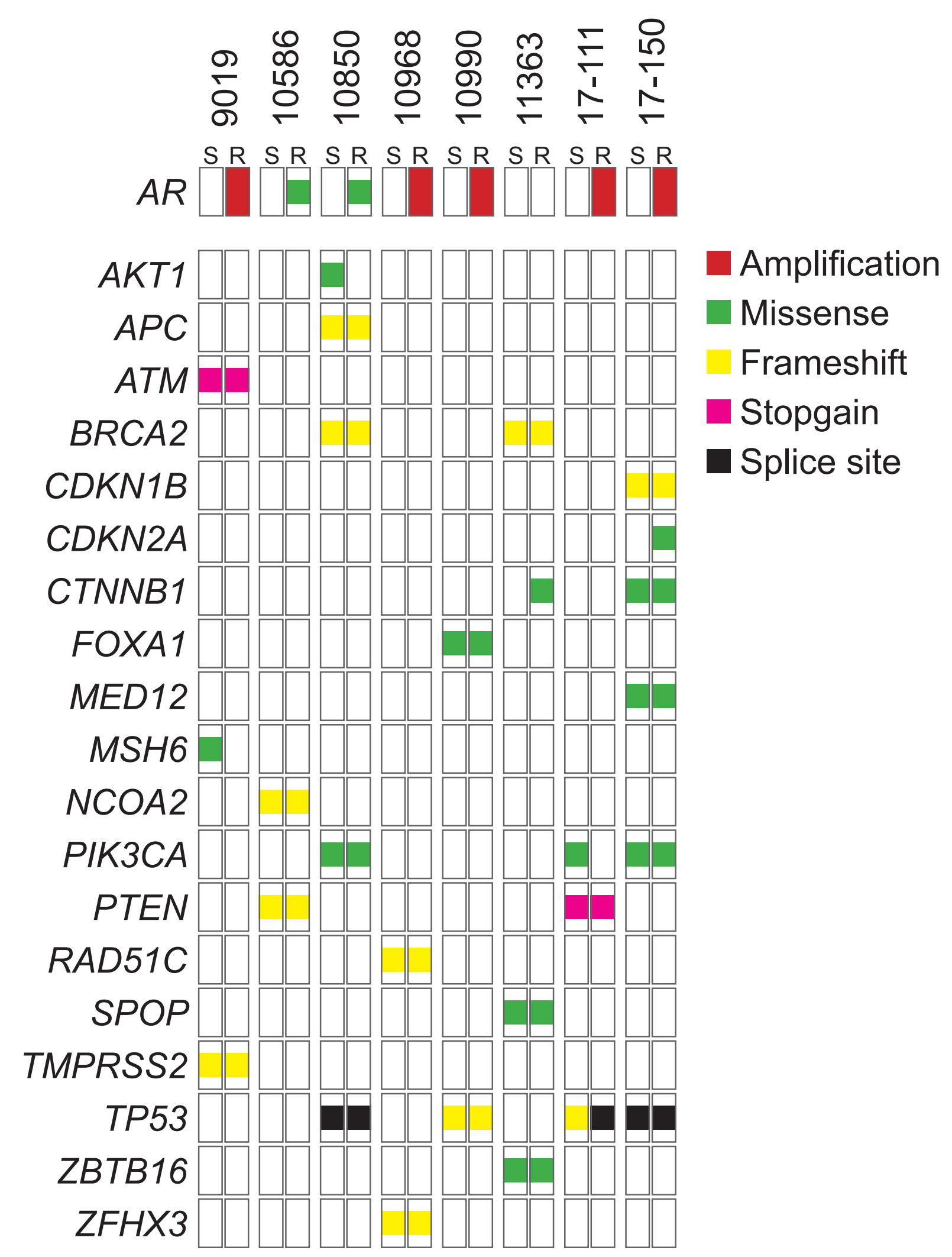


Figure 4. Genomic changes at progression to castration-resistant prostate cancer (CRPC). Oncoprint illustrating similarities between matched castrate-sensitive (S) and castrate-resistant $(\mathrm{R})$ collections, with the exception of the $A R$ gene. Copy number alterations only included for the $A R$ gene. 